\title{
Divergência genética entre clones de cana-de-açúcar usando análise multivariada associada a modelos mistos
}

\section{Multivariate genetic divergence among sugarcane clones by multivariate analysis associated with mixed models}

\author{
Valéria Rosa Lopes ${ }^{1 *}$; Joao Carlos Bespalhok Filho²; Edelclaiton Daros²; \\ Ricardo Augusto Oliveira ${ }^{2}$; Edson Perez Guerra ${ }^{3}$
}

\begin{abstract}
Resumo
Este trabalho teve como objetivo avaliar a divergência genética em clones da cana-de-açúcar usando a metodologia de dispersão gráfica por componentes principais associada aos modelos lineares mistos, identificando quais os genótipos mais divergentes e os mais produtivos de forma mais precisa, para posterior combinação. Foram avaliados 138 clones de cana-de-açúcar da Série RB97 do Programa de Melhoramento Genético de Cana-de-Açúcar da Universidade Federal do Paraná, mais duas variedades padrões, em três ambientes, com duas repetições. Os dois primeiros componentes explicaram 96\% da variação total, sendo suficientes para explicar a divergência encontrada. A característica que mais contribuiu para a divergência genética foi quilograma de brix por parcela (KBP), seguida por brix, massa de 10 colmos (M10) e número de colmos por parcela (NCP). Os clones mais divergentes foram RB975008, RB975112, RB975019, RB975153 e RB975067 e os clones mais produtivos foram RB975269, RB977533, RB975102, RB975317 e RB975038.

Palavras chave: Saccharum officinarum, variabilidade genética, modelos mistos, componentes principais
\end{abstract}

\begin{abstract}
This work had the aim to evaluate the genetic divergence in sugarcane clones using the methodology of graphic dispersion by principal components analysis associated to linear mixed models, indentifying the more divergent and productive genotypes with more precision, for a subsequent combination. 138 sugarcane clones of the RB97 series of the Sugarcane Breeding Program of the Universidade Federal do Parana, more two standard cultivars were evaluated in three environments, with two replications. The two first components explained $96 \%$ of the total variation, sufficiently for explaining the divergence found. The variable that contributed the most to de divergence was kilogram of brix per plot (BKP) followed by brix, mass of 10 stalks and number of stalks per plot. The more divergent sugarcane clones were RB975008, RB975112, RB975019, RB975153 and RB975067 and the more productive clones were RB975269, RB977533, RB975102, RB975317 and RB975038.
\end{abstract}

Key words: Saccharum officinarum, genetic variability, mix models, principal components

${ }^{1}$ Dr. em Agronomia, Companhia de Desenvolvimento do Vale do São Francisco, Brasília, DF. E-mail: agroval@yahoo.com

2 Profs. Drs. Dept ${ }^{\circ}$ de Fitotecnia, Universidade Federal do Paraná, UFPR, Curitiba, PR. E-mail: bespa@ufpr.br; ededaros@ufpr.br; rico@ufpr.br

${ }^{3}$ Prof. Dr. do Dept ${ }^{\mathrm{o}}$ de Agronomia, Universidade Estadual do Centro-Oeste, UNICENTRO, Guarapuava, PR. E-mail: e.guerra@ ufpr.br

* Autor para correspondência 


\section{Introdução}

Alguns autores mencionam que a base genética da cana-de-açúcar é estreita, o que reduz a variabilidade e conseqüentemente pode acarretar perdas de produtividade devido à endogamia (AHMED; OBEID, 2010).

Nesse sentido diferentes estudos têm focado na avaliação da divergência genética entre acessos de bancos de germoplasma e cultivares de canade-açúcar (LIMA et al., 2002; SAJJAD; KHAN, 2009), entretanto, os trabalhos tem sido pouco conclusivos em relação às progênies provenientes desses cruzamentos, nas quais se baseia a seleção de futuras variedades.

Existem diferentes métodos para o estudo da divergência genética, que podem ser adotados de acordo com o objetivo do programa de melhoramento (CRUZ; REGAZZI; CARNEIRO, 2004). Entre eles estão os métodos preditivos que consistem na avaliação de vários caracteres morfoagronômicos e a sua contribuição para o total da variabilidade genética entre os genótipos, usando para isso, métodos aglomerativos e principalmente gráficos de dispersão (Componentes Principais). A metodologia dos componentes principais possui a vantagem de possibilitar a avaliação da importância de cada caractere na variação total, fornecendo parâmetros para o descarte de caracteres que apresentam alta correlação entre si ou apresentam pouca influência na variabilidade total (CRUZ; REGAZZI, 1997; CRUZ; REGAZZI; CARNEIRO, 2004).

Estes métodos já têm se mostrado eficiente na diferenciação de genótipos de cana-de-açúcar (VIANA et al., 1991; KASHIF; KHAN, 2007; SAJJAD; KHAN, 2009). Porém, a alta influência ambiental sofrida por caracteres quantitativos, os mais utilizados na seleção de clones em programas de melhoramento de cana-de-açúcar, torna seus resultados menos precisos quando comparados a outras técnicas.
Nesse sentido a utilização de modelos lineares mistos na análise da divergência genética pode promover resultados mais precisos, pois possui a vantagem de usar valores genotípicos ao invés de valores fenotípicos, promovendo resultados mais precisos quando comparados aos métodos estatísticos convencionais, inclusive na análise da divergência genética (RESENDE, 2006), como verificado em diferentes estudos (OLIVEIRA et al., 2004; ROCHA et al. 2007).

No caso da cana-de-açúcar os modelos lineares mistos têm sido usados para estimar parâmetros genéticos e predizer ganhos (RESENDE, 2002) apresentando melhor índice de eficiência na seleção de genótipos superiores (PEDROZO et al., 2009).

Com base nesses preceitos este trabalho objetivou avaliar a divergência genética em 138 clones de cana-de-açúcar e duas variedades comerciais, com base nos valores genéticos preditos por modelo misto e associados à análise de componentes principais, visando a posterior combinação destes indivíduos em novos cruzamentos.

\section{Material e Métodos}

A análise da divergência genética foi realizada utilizando-se dados provenientes de experimentos de competição de clones promissores da série RB97, da terceira fase de seleção (fase T3), do Programa de Melhoramento Genético de Cana-de-Açúcar da Universidade Federal do Paraná (PMGCA/ UFPR), mais duas variedades padrões RB72454 e RB835486 (Tabela 1).

A avaliação foi realizada no ano de 2005, em três diferentes locais: Estação Experimental de Paranavaí, no município de Paranavaí, 52,5 longitude oeste e $23^{\circ}$ latitude sul; município de Colorado, $52^{\circ}$ longitude oeste e $22,9^{\circ}$ latitude sul; e município de Iguatemi, $52^{\circ}$ longitude e $23,4^{\circ}$ latitude sul, todos no Estado do Paraná. 
Tabela 1. Clones de cana-de-açúcar da fase T3 série RB97 e variedades padrão utilizadas na avaliação da divergência genética.

\begin{tabular}{|c|c|c|c|c|c|c|c|}
\hline 1 & RB975000 & 36 & RB975073 & 71 & RB975177 & 106 & RB975293 \\
\hline 2 & RB975002 & 37 & RB975078 & 72 & RB975179 & 107 & RB975294 \\
\hline 3 & RB975004 & 38 & RB975079 & 73 & RB975183 & 108 & RB975298 \\
\hline 4 & RB975005 & 39 & RB975080 & 74 & RB975184 & 109 & RB975299 \\
\hline 5 & RB975006 & 40 & RB975081 & 75 & RB975185 & 110 & RB975317 \\
\hline 6 & RB975007 & 41 & RB975083 & 76 & RB975188 & 111 & RB975323 \\
\hline 7 & RB975008 & 42 & RB975086 & 77 & RB975200 & 112 & RB975329 \\
\hline 8 & RB975010 & 43 & RB975087 & 78 & RB975202 & 113 & RB975337 \\
\hline 9 & RB975013 & 44 & RB975088 & 79 & RB975204 & 114 & RB975338 \\
\hline 10 & RB975015 & 45 & RB975089 & 80 & RB975205 & 115 & RB975339 \\
\hline 11 & RB975019 & 46 & RB975090 & 81 & RB975206 & 116 & RB975345 \\
\hline 12 & RB975021 & 47 & RB975092 & 82 & RB975207 & 117 & RB975350 \\
\hline 13 & RB975022 & 48 & RB975094 & 83 & RB975211 & 118 & RB975353 \\
\hline 14 & RB975024 & 49 & RB975100 & 84 & RB975212 & 119 & RB976300 \\
\hline 15 & RB975026 & 50 & RB975101 & 85 & RB975217 & 120 & RB976306 \\
\hline 16 & RB975027 & 51 & RB975102 & 86 & RB975219 & 121 & RB976310 \\
\hline 17 & RB975029 & 52 & RB975103 & 87 & RB975221 & 122 & RB976311 \\
\hline 18 & RB975031 & 53 & RB975108 & 88 & RB975224 & 123 & RB976315 \\
\hline 19 & RB975032 & 54 & RB975111 & 89 & RB975228 & 124 & RB976317 \\
\hline 20 & RB975033 & 55 & RB975112 & 90 & RB975235 & 125 & RB976319 \\
\hline 21 & RB975038 & 56 & RB975114 & 91 & RB975238 & 126 & RB976320 \\
\hline 22 & RB975045 & 57 & RB975115 & 92 & RB975241 & 127 & RB976324 \\
\hline 23 & RB975046 & 58 & RB975151 & 93 & RB975243 & 128 & RB976328 \\
\hline 24 & RB975047 & 59 & RB975153 & 94 & RB975244 & 129 & RB976331 \\
\hline 25 & RB975048 & 60 & RB975157 & 95 & RB975249 & 130 & RB976336 \\
\hline 26 & RB975049 & 61 & RB975163 & 96 & RB975253 & 131 & RB976338 \\
\hline 27 & RB975051 & 62 & RB975164 & 97 & RB975256 & 132 & RB976339 \\
\hline 28 & RB975053 & 63 & RB975165 & 98 & RB975269 & 133 & RB976341 \\
\hline 29 & RB975055 & 64 & RB975166 & 99 & RB975270 & 134 & RB977529 \\
\hline 30 & RB975057 & 65 & RB975169 & 100 & RB975279 & 135 & RB977533 \\
\hline 31 & RB975061 & 66 & RB975170 & 101 & RB975282 & 136 & RB977534 \\
\hline 32 & RB975067 & 67 & RB975172 & 102 & RB975285 & 137 & RB977543 \\
\hline 33 & RB975068 & 68 & RB975173 & 103 & RB975286 & 138 & RB977619 \\
\hline 34 & RB975069 & 69 & RB975174 & 104 & RB975289 & 139 & RB72454 \\
\hline 35 & RB975070 & 70 & RB975175 & 105 & RB975290 & 140 & RB835486 \\
\hline
\end{tabular}

Fonte: Elaboração dos autores.

O delineamento experimental utilizado foi o de blocos aumentados ou blocos de Federer (FEDERER, 1956) com duas repetições (campo “A" e "B"). Cada bloco foi composto por 18 clones promissores da série RB97 mais duas variedades, RB72454 e RB835486 utilizadas como padrões. As unidades experimentais foram compostas por parcelas contendo dois sulcos de 5 metros, espaçados entre si por 1,40 m na localidade de Paranavaí, e
1,10 m nas demais localidades. O plantio dos clones foi realizado utilizando-se toletes contendo três gemas, de forma que em cada unidade experimental foram distribuídos 60 toletes, obtendo-se 18 gemas $\mathrm{m}^{-1}$ de sulco.

Optou-se por utilizar nesta análise apenas os clones cujos genitores eram conhecidos (cruzamentos bi-parentais) e repetidos nos três 
locais, para efeito de comparação dos dados. Dessa forma, foram utilizados ao todo 138 clones, mais as duas variedades padrões.

As avaliações foram feitas em cana-soca e os caracteres avaliados foram aqueles utilizados na seleção de clones na fase T3: número de colmos por parcela (NCP); massa de 10 colmos (M10); brix e produção de brix por parcela (KBP): expressa em quilograma, calculado pela fórmula: $\mathrm{KBP}=(\mathrm{M} 10 \mathrm{x}$ NCP x Brix) $/ 10^{-3}$.

A estimativa de componentes de variância e predição de valores genéticos foi realizada usando o procedimento de melhor predição linear não tendencioso/máxima verossimilhança restrita (REML/BLUP), usando o programa SelegenREML/BLUP (RESENDE, 2006).

Para a análise de Deviance em cada ambiente foi utilizado o seguinte modelo estatístico, segundo Resende (2006):

$$
\mathrm{y}=\mathrm{Xf}+\mathrm{Zg}+\mathrm{Wb}+\mathrm{e}
$$

Em que: y: vetor de dados; f: vetor dos efeitos assumidos como fixos; g: vetor dos efeitos genotípicos (assumidos como aleatórios); b: vetor dos efeitos ambientais de blocos (assumidos como aleatórios); e: vetor de erros ou resíduos (aleatórios); e $\mathrm{X}, \mathrm{Z}$ e $\mathrm{W}$, representam as matrizes de incidência para os efeitos de f, $\mathrm{g}, \mathrm{e} \mathrm{b}$, respectivamente.

Para a análise conjunta dos ambientes o modelo utilizado foi:

$$
\mathrm{y}=\mathrm{Xf}+\mathrm{Zg}+\mathrm{Wb}+\mathrm{Ti}+\mathrm{e}
$$

Em que: y: vetor de dados; f: vetor dos efeitos assumidos como fixos (média de locais); g: vetor dos efeitos genotípicos (assumidos como aleatórios); b: vetor dos efeitos ambientais de blocos (assumidos como aleatórios); i: vetor dos efeitos da interação genótipo x ambiente (aleatórios), e: vetor de erros ou resíduos (aleatórios); e X, Z, W e T = representam as matrizes de incidência para os efeitos de $f, g, b$, e i, respectivamente.
Aanálise de deviance (ANADEV), utilizada neste trabalho, é similar à análise de variância (ANOVA), porém permite a análise de dados desbalanceados com maior precisão dos componentes da variância e acurácia dos valores genéticos em relação à ANOVA, pois trabalha com valores genotípicos e não fenotípicos. Além disso, o método REML permite lidar com a situação de tratamentos correlacionados, comum no melhoramento genético, o que não acontece com a ANOVA que supõem a independência dos erros a dos efeitos de tratamento (RESENDE, 2006).

A divergência genética foi obtida pela técnica de análise multivariada, sendo adotado o método análise de componentes principais. A análise genética para caracteres múltiplos baseou-se nas seguintes premissas: a) Os efeitos dos tratamentos considerados como aleatórios; b) Valores genotípicos ao invés de valores fenotípicos; c) Considerou o desbalanceamento dos dados; e d) Análise da divergência juntamente com a predição dos valores genotípicos, pois os valores genotípicos são preditos com diferentes precisões, sendo considerado na análise (RESENDE, 2007).

A análise de componentes principais foi realizada baseada nos valores genotípicos preditos pelo procedimento BLUP, usando as correlações entre as variáveis e os componentes principais (RESENDE, 2007). O gráfico foi montado considerando os três primeiros escores dos componentes principais, por representarem mais de $80 \%$ da variação total presente (CRUZ; CARNEIRO, 2004).

Para agrupamento dos indivíduos, dentro dos gráficos de dispersão formados pelos componentes principais, foi utilizado o agrupamento de Tocher, também realizado no programa Selegen.

\section{Resultados e Discussão}

Análise de variância

A análise de deviance revelou que há diferenças significativas entre os genótipos (clones da série 
RB97), para quase todos os caracteres avaliados, em todos os locais (ambientes), para o nível de significância de $1 \%(\mathrm{P}<0.01)$, exceto para o caractere KBP, em Paranavaí, que o efeito de genótipos foi significativo a $5 \%(\mathrm{P}<0.05)$ e em Colorado que não foi significativo (Tabela 2). Esse resultado indica que há variabilidade genética no material estudado, sendo possível o estudo da divergência, e que estes materiais poderiam ser utilizados em cruzamentos com o objetivo de gerar variabilidade nas gerações segregantes.

Tabela 2. Resultados da análise de Deviance (ANADEV), individual e conjunta, dos 138 clones de cana-de-açúcar da série RB97, mais as variedades padrões RB835486 e RB72454 para os quatro caracteres avaliados em três ambientes no Estado do Paraná.

\begin{tabular}{cccccc}
\hline \multirow{2}{*}{ Local } & \multirow{2}{*}{ Efeitos } & \multicolumn{4}{c}{ Caracter } \\
\cline { 3 - 5 } & & $\mathbf{M 1 0}{ }^{(1)}$ & NCP & Brix & KBP \\
\hline Colorado & \multirow{2}{*}{ Genótipo } & $21,56^{* *(2)}$ & $11,49^{* *}$ & $10,23^{* *}$ & $0,72^{\text {ns }}$ \\
Paranavaí & & $18,91^{* *}$ & $19,58^{* *}$ & $59,93 * *$ & $3,55^{*}$ \\
Iguatemi & $21,99^{* *}$ & $61,06^{* *}$ & $72,99 * *$ & $16,16^{* *}$ \\
\hline \multirow{2}{*}{ Conjunta } & Genótipo & $56,29^{* *}$ & $52,92^{* *}$ & $57,25 * *$ & $18,91^{* *}$ \\
& Interação genótipo x ambiente & $5,14^{*}$ & $19,77^{* *}$ & $22,63 * *$ & $3,54 *$ \\
\hline
\end{tabular}

Massa de dez colmos - M10; número de colmos por parcela - NCP; e quilograma de brix por parcela - KBP

Valores de Qui-quadrado $\left(\mathrm{X}^{2}\right)$ tabelado: 2,71 e 6,63 para os níveis de significância de $5 \%(*)$ e $1 \%(* *)$ de probabilidade, respectivamente, e ${ }^{\left({ }^{\mathrm{ns}}\right)}$ não significativo.

Fonte: Elaboração dos autores.

Também houve efeito significativo da interação genótipos-ambientes na análise conjunta, para todos os caracteres avaliados (Tabela 2), mesmo com o uso de valores genotípicos, que minimizam o efeito ambiental.

Os clones mais produtivos de acordo com o valor genotípico em Colorado foram RB975079, RB975290, RB975299, RB975269, RB72454; em Paranavaí foram RB975102, RB975153, RB975317, RB976339, RB975244; e em Iguatemi foram RB975285, RB975279, RB975175, RB975033, RB977619. Já os genótipos RB975269, RB977533, RB975102, RB975317 se destacaram, com maiores valores genotípicos, no resultado da análise conjunta (dados não mostrados).

\section{Divergência genética}

Segundo Cruz, Regazzi e Carneiro (2004) a metodologia dos componentes principais é considerada eficiente quando os dois primeiros componentes explicam pelo menos $80 \%$ da variação total encontrada. Em todos os ambientes os dois primeiros componentes explicaram mais de $80 \%$ da variação total; $98,07 \%, 96,84 \%$ e $94,98 \%$ para Colorado, Paranavaí e Iguatemi, respectivamente, como pode ser observado na tabela 3. Esses resultados indicam que a metodologia foi satisfatória no estudo da divergência dos clones de cana-deaçúcar (CRUZ; REGAZZI; CARNEIRO, 2004). 
Tabela 3. Autovalores e coeficientes de ponderação dos 138 clones de cana-de-açúcar e duas variedades padrões nos três ambientes e análise conjunta, no Estado do PR.

\begin{tabular}{|c|c|c|c|c|c|c|}
\hline \multirow{2}{*}{ Local } & \multirow{2}{*}{$\mathbf{C P}$} & \multirow{2}{*}{$\begin{array}{c}\text { Autovalores } \\
\text { Acumulados (\%) }\end{array}$} & \multicolumn{4}{|c|}{ Coeficientes de ponderação associados à: } \\
\hline & & & M10 $^{(1)}$ & NCP & Brix & KBP \\
\hline \multirow{4}{*}{ Colorado } & $\mathrm{CP}_{1}$ & 98,07 & $-4,760$ & $-3,894$ & $-1,452$ & 5,315 \\
\hline & $\mathrm{CP}_{2}$ & 99,56 & $-0,399$ & $-0,495$ & 0,826 & 0,692 \\
\hline & $\mathrm{CP}_{3}^{2}$ & 99,98 & 1,009 & 0,194 & $-0,076$ & 0,018 \\
\hline & $\mathrm{CP}_{4}^{3}$ & 100 & 0,083 & 1,014 & 0,043 & 0,011 \\
\hline \multirow{4}{*}{ Paranavaí } & $\mathrm{CP}_{1}^{4}$ & 96,84 & $-4,787$ & $-4,801$ & $-1,730$ & 6,102 \\
\hline & $\mathrm{CP}_{2}$ & 99,31 & $-0,817$ & $-0,226$ & 0,732 & 0,2204 \\
\hline & $\mathrm{CP}_{3}^{2}$ & 99,98 & 0,052 & $-0,582$ & 0,358 & 0,955 \\
\hline & $\mathrm{CP}_{4}$ & 100 & 0,042 & 0,996 & 0,048 & 0,027 \\
\hline \multirow{4}{*}{ Iguatemi } & $\mathrm{CP}_{1}$ & 94,98 & $-4,045$ & $-4,202$ & $-1,056$ & 5,819 \\
\hline & $\mathrm{CP}_{2}$ & 99,36 & $-0,585$ & $-0,136$ & 0,875 & 0,065 \\
\hline & $\mathrm{CP}_{3}^{2}$ & 99,98 & $-0,013$ & $-0,959$ & 0,254 & 1,261 \\
\hline & $\mathrm{CP}_{4}$ & 100 & 0,026 & 0,975 & 0,036 & 0,032 \\
\hline \multirow{4}{*}{ Análise Conjunta } & $\mathrm{CP}_{1}^{4}$ & 96,62 & $-5,231$ & $-5,078$ & $-1,725$ & 6,204 \\
\hline & $\mathrm{CP}_{2}$ & 99,41 & $-0,974$ & $-0,706$ & 0,700 & 0,694 \\
\hline & $\mathrm{CP}_{3}^{2}$ & 99,98 & 0,102 & $-0,655$ & 0,207 & 1,045 \\
\hline & $\mathrm{CP}_{4}$ & 100 & 0,044 & 0,991 & 0,037 & 0,032 \\
\hline
\end{tabular}

(1) M10 - massa de 10 colmos; NCP - número de colmos por parcela; e KBP - quilograma de brix por parcela.

Fonte: Elaboração dos autores.

A metodologia dos componentes principais ainda permite avaliar a importância dos caracteres sobre a variação total disponível entre os genótipos estudados, permitindo a exclusão dos que apresentam pouca contribuição na discriminação do material avaliado, com base nos valores do coeficiente de ponderação (CRUZ; REGAZZI; CARNEIRO, 2004).

Com base no princípio de que a importância relativa dos componentes principais decresce do primeiro para o último, tem-se que os caracteres que mais contribuem para a divergência são aqueles com maiores coeficientes de ponderação nos dois primeiros componentes principais, enquanto os últimos componentes são responsáveis pela explicação de uma fração mínima da variância total disponível e apresentam o maior valor absoluto no último componente principal (CRUZ; REGAZZI; CARNEIRO, 2004).

Sendo assim, para todos os locais o caractere que menos contribuiu para a divergência genética foi NCP $(1,011 ; 0,996 ; 0,974$, para Colorado Paranavaí e Iguatemi, respectivamente), enquanto o que mais contribuiu foi $\operatorname{KBP}(5,315 ; 6,102 ; 5,819$, para Colorado Paranavaí e Iguatemi, respectivamente). Em ordem decrescente, os caracteres que mais contribuíram para a divergência, em todos os locais, foram KBP, Brix, M10 e NCP (Tabela 3).

A baixa contribuição do caractere número de colmos por parcela para a divergência genética total pode estar no fato de haver uma estabilização do canavial com aproximadamente 12 colmos por metro linear após seis a oito meses do perfilhamento, independendo do genótipo (MACHADO et al., 1982). Para Leite, Peternelli e Barbosa (2006) o número de colmos é o mais estável dos caracteres e, também, muito importante para predizer ganhos esperados com seleção, ou seja, apresenta pouca variação em relação ao ambiente.

Este resultado foi diferente ao obtido por Silva et al. (2005), trabalhando com a série RB91 e avaliando cinco caracteres, em que o caractere número de colmos por parcela (NCP), foi o que mais contribuiu para a divergência genética, enquanto o caractere que menos contribuiu para a divergência total foi massa média de 10 colmos (M10), porém os 
autores não mencionaram o possível motivo para os resultados encontrados. $\mathrm{O}$ fato de o caractere $\mathrm{KBP}$ ter sido o que mais contribuiu para a divergência no presente estudo pode estar associado ao fato de este ser estimado a partir dos demais, concentrando assim toda a divergência.

Embora o caractere Brix não tenha sido o caractere que mais contribuiu para a divergência genética total, a sua inclusão nessa análise é fundamental, pois este é um dos caracteres de maior importância para a cultura da cana-de-açúcar, bem como a massa média de 10 colmos, pois são responsáveis pela produção de açúcar por área, devendo ser mantidos em futuras análises.

\section{Gráficos de dispersão}

Os grupos formados nos gráficos de dispersão (Figuras 1 e 2 ) são os mesmos encontrados pela análise de agrupamento de Tocher, e a sua visualização foi possível devido ao fato dos mesmos encontrarem-se distintos nos gráficos, exceto na análise conjunta onde os grupos 1, 2 e 3 ficaram muito dispersos.

O número de grupos formados foi relativamente pequeno, sendo que a maioria dos indivíduos (92 a 96\%) permaneceu em apenas um grupo, enquanto um número reduzido de indivíduos (4 a 8\%) formou os demais grupos (Figuras 1 e 2). Estes resultados são semelhantes aos encontrados por Silva et al. (2005) que, estudando os clones da série RB91, encontraram um número reduzido de grupos e estes também apresentaram maior concentração dos clones no primeiro grupo ( 84 a $44 \%$ ) nos dois ambientes estudados, resultados estes, segundo os autores, atribuídos à baixa divergência genética entre os clones da referida série.

Deve-se ressaltar que em etapas mais avançadas dos programas de melhoramento de cana-deaçúcar já houve grande quantidade de indivíduos descartados e que a seleção é voltada para caracteres de interesse. Desta forma, os indivíduos da Fase T3 são mais próximos fenotipicamente devido às etapas anteriores de seleção, que alteram a média genotípica na direção desejada, o que pode explicar parcialmente o pequeno número de grupos formados. Ainda, ressalta-se que parte dos genótipos estudados é de irmãos germanos, o que torna os resultados em relação à divergência mais importante, pois enfatiza a existência de variabilidade. Uma possibilidade no estudo da divergência seria estimar essa nas fases iniciais do programa, quando a seleção ainda não foi realizada, porém sem dados de repetição.

Como pode ser observado na Figura 1 o número de grupos de similaridade formados e a composição dos mesmos variou de acordo com o ambiente em que foi avaliada a divergência. Em Colorado os indivíduos mais divergentes foram os do Grupo 2 (RB976306 e RB975079) em relação ao Grupo 4 (RB975217), ou ainda o Grupo 3 (RB835486 e RB72454) em relação ao Grupo 2.

Figura 1. Dispersão gráfica dos escores em relação aos dois primeiros componentes principais, CP1 e CP2, dos 138 clones de cana-de-açúcar mais as variedades padrões RB72454 e RB 835486, em Colorado (A), Paranavaí (B) e Iguatemi (C) e grupos formados de acordo com o agrupamento de Tocher.
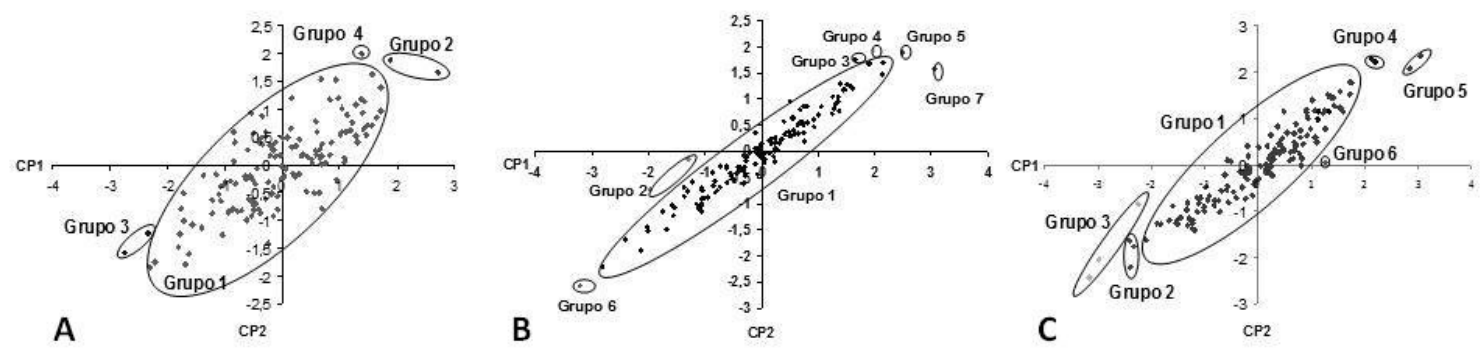

Fonte: Elaboração dos autores. 
Em Paranavaí houve a maior dispersão dos dos grupos 2 (RB975163 e RB755088) e 6 clones, o que dificultou a formação de grupos maiores. Ao todo foram formados sete grupos de similaridade, sendo os mais distantes os indivíduos

(RB975102) em relação aos indivíduos dos grupos 3 (RB977619), 4 (RB975070), 5 (RB835486) e 7 (RB975165) (Figura 2).

Figura 2. Dispersão gráfica dos escores em relação aos dois primeiros componentes principais, CP1 e CP2, dos 138 clones de cana-de-açúcar mais as variedades padrões RB72454 e RB835486, nos três ambientes (Análise Conjunta) e grupos formados de acordo com o agrupamento de Tocher.

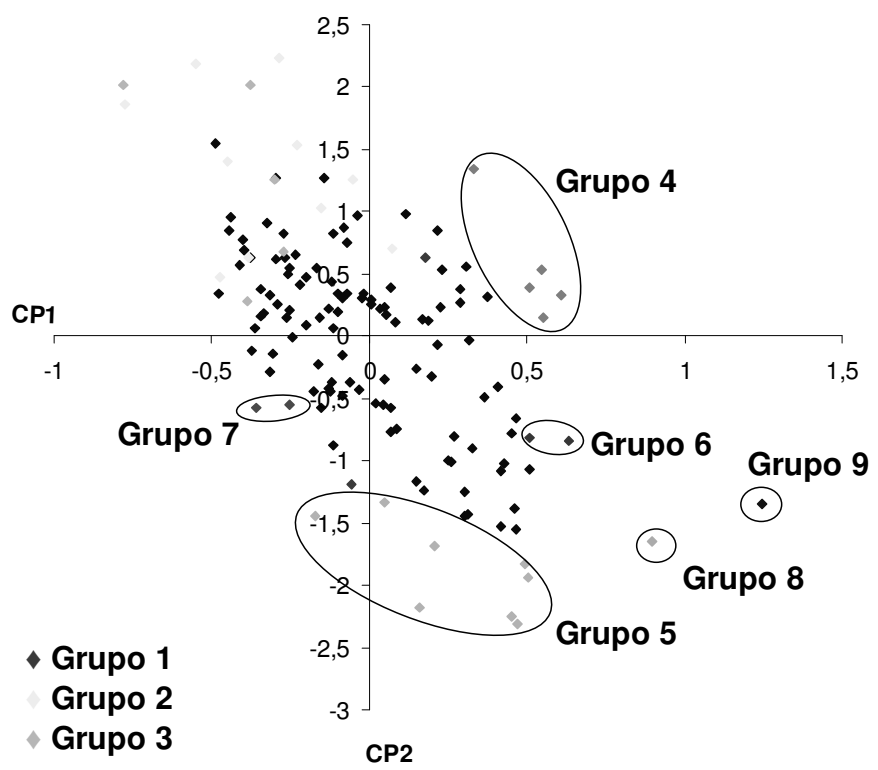

Fonte: Elaboração dos autores.

Foram formados seis grupos de similaridade em Iguatemi, sendo os mais divergentes os indivíduos presentes nos grupos 2 (RB975089, RB975008 e RB975087) e 3 (RB975299, RB72454 e RB975114) em relação aos indivíduos presentes nos grupo 4 (RB975153 e RB975031), 5 (RB975285 e RB975019) e 6 (RB975090).

Uma observação importante é que genótipos provenientes de um mesmo cruzamento permaneceram em grupos divergentes, como é o caso, em Colorado, dos clones RB975217 (Grupo 4) e RB975179 (Grupo 1) ambos provenientes dos cruzamentos dos genótipos RB835486 com RB855113; ou ainda indivíduos de cruzamentos diferentes permaneceram no mesmo grupo e muito próximos geneticamente, como os clones RB975027 e RB975169, provenientes dos cruzamentos das variedades RB855206 com IAC49-131 e RB825548 com RB835486, respectivamente. Usando esta comparação novamente é possível perceber que ainda há variabilidade genética entre os clones estudados, mesmo com o pequeno número de grupos formados, evidenciando a complexidade genética da cana-de-açúcar e a alta heterosigose.

Com o objetivo de reduzir a influência do ambiente, também foi realizada a análise conjunta dos dados utilizando os componentes principais (Figura 2). Os dois primeiros componentes principais explicaram mais de $80 \%$ da variação total $(99,41 \%)$, para todos os caracteres em estudo, como pode ser observado pelos autovalores acumulados, sendo que KBP foi o caracter que mais contribuiu 
para a divergência enquanto o que menos contribuiu foi NCP (Tabela 3), concordando com os demais resultados.

Os resultados em relação ao gráfico de dispersão, na análise conjunta, são semelhantes àqueles encontrados nos demais (Figura 2). Percebe-se pelo gráfico que a dispersão dos dados é maior em relação à dispersão nos ambientes separados, formando nove grupos ao todo, não sendo possível visualizar de forma clara os grupos 1, 2 e 3 . Os grupos que apresentaram os indivíduos mais divergentes nessa análise foram: grupo 4 (RB975088, RB975005, RB975087, RB975070 e RB975032), grupo 5 (RB975038, RB975244, RB975102, RB976339, RB975317, RB977533 e RB975083), grupo 6 (RB975114 e RB975067), grupo 7 (RB975019 e RB975153), grupo 8 (RB975112) e grupo 9 (RB975008).

A formação de vários grupos mostra novamente a existência de variabilidade na população, concordando com os resultados de Ferreira et al. (2005) que comparando diferentes tipos de cruzamento, encontraram baixos níveis de depressão dentro das famílias, obtidas por auto-fecundação da variedade RB72454, resultado este atribuído à provável alta freqüência de alelos favoráveis, indicando que este fator é tão importante quanto a heterose.

Como na análise foram utilizados poucos caracteres, uma possibilidade seria a utilização de um maior número de características com o objetivo de tornar os resultados mais precisos. Corroborando comessainformação, Silva etal.(2001)mencionaram que a divergência entre acessos retrata somente a variabilidade existente nas características avaliadas para sua estimação, não permitindo extrapolações para outros caracteres. No entanto é preciso analisar a relevância da utilização dos caracteres usados, que devem possuir menor resposta às condições ambientais (não quantitativos), porém devem ser caracteres de importância para o melhoramento genético, uma vez que são eles que determinam se um genótipo se tornará uma variedade ou não.

\section{Conclusão}

A metodologia de componentes principais associada aos modelos lineares mistos mostrou que há variabilidade genética entre os clones de cana-deaçúcar estudados, em todos os ambientes. Os clones mais divergentes foram RB975008, RB975112, RB975019, RB975153 e RB975067 e os clones mais produtivos foram RB975269, RB977533, RB975102, RB975317 e RB975038. O caractere que mais contribuiu para a divergência genética total foi quilograma de brix por parcela, e o que menos contribuiu foi o caractere número de colmos por parcela. Os clones mais produtivos e mais divergentes podem ser combinados em novas etapas de cruzamentos e seleções visando à obtenção de genótipos mais produtivos e com variabilidade.

\section{Referências}

AHMED, A. O.; OBEID, A. Genetic divergence among sugarcane genotypes (Saccharum spp.) for cane yield attributes and quality determinants. African Journal of Agricultural Research, Nigéria, v. 5, n. 16, p. 2103-2107, 2010.

CRUZ, C. D.; CARNEIRO, P. C. S. Modelos biométricos aplicados ao melhoramento genético. Viçosa: Editora UFV, 2003. 585 p.

. Modelos biométricos aplicados ao melhoramento genético. 2. ed. Viçosa: Editora UFV, 1997. 390 p.

CRUZ, C. D.; REGAZZI, A. J.; CARNEIRO, P. C. S. Modelos Biométricos aplicados ao melhoramento genético. 3. ed. Viçosa: Editora UFV, 2004. 460 p.

FEDERER, W. T. Augmented (or hoonuiaku) designs. Hawaian Planters Record, Hawai, v. 55, n. 2, p. 191-208, 1956.

FERREIRA, F. M.; BARBOSA, M. H. P.; CASTRO, R. D.; PATERNELLI, L. A.; CRUZ, C. D. Effects of inbreeding on the selection of sugar cane clones. Crop Breeding and Applied Biotechnology, Viçosa, MG, v. 5, n. 2, p. 174-182, 2005.

KASHIF, M.; KHAN, F. A. Divergence in sugarcane (Saccharum officinarum L.) based on yield and quality traits. Pakistan Journal of Botany, Pakistan, v. 39, n. 5, p. 1559-1563, 2007. 
LEITE, M. S. O.; PETERNELLI, L. A.; BARBOSA, M. H. P. Effects of plot size on the estimation of genetic parameters in sugarcane families. Crop Breeding and Applied Biotechnology, Viçosa, MG, v. 6, n. 1, p. 40-46, 2006.

LIMA, M. L. A.; GARCIA, A. A. F.; OLIVEIRA, K. M.; MATSUOKA, S.; SOUZA JÚNIOR, A. H. de; C. L.; SOUZA, A. P. de. Analysis of genetic similarity detected by AFLP and coefficient of parentage among genotypes of sugar cane (Saccharum spp.). Theoretical and Applied Genetics, New York, v. 104, n. 1, p. 3-38, 2002.

MACHADO, E. C.; PEREIRA, A. R.; FAHL, J. L.; ARRUDA, H. V.; CIONE, J. Índices biométricos de duas variedades de cana-de-açúcar. Pesquisa Agropecuária Brasileira, Brasília, v. 17, n. 9, p. 1321-1329, 1982.

OLIVEIRA, V. R.; RESENDE, M.D. V.; NASCIMENTO, C. E. de S.; DRUMOND, M. A.; SANTOS, C. A. F. Variabilidade genética de procedências e progênies de umbuzeiro via metodologia de modelos lineares mistos (REML/BLUP). Revista Brasileira de Fruticultura, Jaboticabal, v. 26, n. 1, p. 53-56, 2004.

PEDROZO, C. A.; BENITES, F. R. G.; BARBOSA, M. H. P.; RESENDE, M. D. V.; SILVA, F. Eficiência de índices de seleção utilizando a metodologia REML/ BLUP no melhoramento da cana-de-açúcar. Scientia Agraria, Curitiba, v. 10, n. 1, p. 31-36, 2009.

RESENDE, M. D. V. Genética, biométrica e estatística: no melhoramento de plantas perenes. Brasília: Embrapa Informação Tecnológica, 2002. 975 p.

O software Selegen Reml/Blup. Campo Grande: Embrapa Informação Tecnológica, 2006. 299 p.

. Selegen-Reml/Blup: sistema estatístico e seleção genética computadorizada via modelos lineares mistos. Colombo: Embrapa Florestas, 2007. 359 p.
ROCHA, M. G. B.; PIRES, I. E.; ROCHA, R. B.; XAVIER, A.; CRUZ, C. D. Seleção de genitores de Eucalyptus grandis e de Eucalyptus urophylla para produção de híbridos interespecíficos utilizando REML/ BLUP e informação de divergência genética. Revista Árvore, Viçosa, MG, v. 31, n. 6, p. 977-987, 2007.

SAJJAD, K.; KHAN, F. A. Genetic Diversity among Sugarcane Cultivars in Pakistan. Journal of Agricultural \& Environmental Sciences, Pakistan, v. 6, n. 6, p. 730736, 2009.

SILVA, C. M.; GONÇALVES-VIDIGAL, M. C.; VIDIGAL FILHO, P. S.; SCAPIM, C. A.; DAROS, E.; SILVÉRIO, L. Genetic diversity among sugarcane clones (Saccharum spp.). Acta Scientiarum Agronomy, Maringá, v. 27, n. 2, p. 315-319, 2005.

SILVA, D. J. H.; COSTA, C. P.; CRUZ, C. D.; CASALI, V. W. D.; DIAS, L. A. S. Stability of genetic divergence among eggplant accesses in three stages of development. Crop Breeding and Applied Biotechnology, Viçosa, MG, v. 1, n. 2, p. 135-143, 2001.

VIANA, J. M. S.; CARDOSO, A. A.; CRUZ, C. D.; REGAZZI, A. J.; DEL GIÚDICE, R. M. Genetic divergence in sugarcane (Saccharum spp.) clones. Revista Brasileira de Genética, Ribeirão Preto, v. 14, n. 3, p. 753-763, 1991. 